\title{
ALGEBRAS WITH UNCONDITIONAL ORTHOGONAL BASES
}

\author{
TAQDIR HUSAIN ${ }^{1}$ AND SALEEM WATSON
}

\begin{abstract}
This is a continuation of our study of topological algebras with orthogonal Schauder bases. In the previous paper, the structure of closed ideals was determined and it was shown that the closed-maximal ideal space is homeomorphic with the discrete space of positive integers. Here it is shown that the space of all maximal ideals equipped with the hull-kernel topology is homeomorphic with the Stone-Cech compactification of natural numbers. Among other results it is also proved that the intersection of dense maximal ideals is isomorphic with the topological dual of the algebra under certain conditions.
\end{abstract}

1. Introduction. Let $\boldsymbol{A}$ be a complete LC-algebra with an unconditional orthogonal Schauder basis. In a previous paper [3] we studied the structure of the closed ideals of such algebras and we showed that $\mathbf{M}(A)$, the closed maximal ideal space of $A$, is homeomorphic to the discrete space of positive integers, $\mathbf{N}$. In this paper we study the space $\mathbf{M}^{\#}(A)$ of all maximal ideals of $A$ equipped with the hull-kernel topology. We show that $\mathbf{M}^{\#}(A)$ is homeomorphic to $\beta \mathbf{N}$, the Stone-Čech compactification of $\mathbf{N}$ (Theorem 1). Our particular method of defining this homeomorphism enables us to characterize an ideal which is the intersection of dense maximal ideals of $A$ (Theorem 2) and we show among other results that under certain conditions such an ideal, as linear space, is isomorphic to $A^{\prime}$, the dual space of $A$ (Theorem 3).

Throughout this paper $A$ will denote a Hausdorff complete LC-algebra with identity $e$ and $\left\{x_{n}\right\}$ is an unconditional (cf. [1]) orthogonal (i.e. $x_{i} x_{j}=\delta_{i j} x_{i}$, cf. [3]) Schauder basis [1] or [5] in $A$. For some properties and examples of such algebras see [3]. For general definitions regarding LC-algebras see [6], and regarding basis see Day [1] or Singer [5].

1. The space $M^{\#}(A)$. If $\left\{x_{n}\right\}$ is a Schauder basis in a linear space $E$, then for each $x \in E$ there is a unique sequence $\left\{\alpha_{n}\right\}$ of scalars such that $x=\sum \alpha_{n} x_{n}$ and each $x_{n}^{*}(x)=\alpha_{n}$ is a continuous linear functional on $A$. Now we begin by proving some simple properties of $A$.

LEMMA 1. The following properties hold:

(a) For any bounded seqeunce $\left\{\alpha_{n}\right\}$ of scalars, $\sum_{n=1}^{\infty} \alpha_{n} x_{n}$ converges in $A$.

(b) If $\left\{x_{n}^{*}(x)\right\}$ is bounded away from zero, then $x$ is invertible.

Received by the editors June 4, 1979, and, in revised form, August 17, 1979.

AMS (MOS) subject classifications (1970). Primary 46H10, 46H15, 46H20.

Key words and phrases. Topological algebras, orthogonal Schauder bases, maximal ideal space.

'This work was supported by an N.R.C. grant. 
(c) If $x=\Sigma \alpha_{n} x_{n} \in A$, then $|x| \equiv \Sigma\left|\alpha_{n}\right| x_{n}$ and $\bar{x} \equiv \Sigma \bar{\alpha}_{n} x_{n}$ both converge in $A$, where $\left|\alpha_{n}\right|$ is the absolute value and $\bar{\alpha}_{n}$ the complex conjugate of the complex number $\alpha_{n}$.

ProOr. (a) follows from the fact that $e=\Sigma x_{n} \in A[3]$ and $\left\{x_{n}\right\}$ is unconditional, hence bounded multiplier convergent [1].

(b) follows from (a) and the fact that $\left(\sum \alpha_{n} x_{n}\right)\left(\sum \beta_{n} x_{n}\right)=\Sigma \alpha_{n} \beta_{n} x_{n}$ (see [3]).

(c) The sequence $\beta_{n}=\operatorname{sgn} \bar{\alpha}_{n}$ is bounded. It follows from the unconditionality of the basis $\left\{x_{n}\right\}$ that $\sum \beta_{n} \alpha_{n} x_{n}=\Sigma\left|\alpha_{n}\right| x_{n} \in A$. The other part is proved similarly.

LEMMA 2. If $I$ is an ideal of $A$ and $x \in I$, then $|x| \in I$ and $\bar{x} \in I$.

Proof. We have $x=\Sigma \alpha_{n} x_{n} \in I$ for some sequence $\left\{\alpha_{n}\right\}$. The sequence $\beta_{n}=$ sgn $\bar{\alpha}_{n}$ is bounded; hence by Lemma 1 (a) $y=\Sigma \beta_{n} x_{n} \in A$. But, $x y \in I$ and $x y=\left(\sum \alpha_{n} x_{n}\right)\left(\sum \beta_{n} x_{n}\right)=\Sigma \alpha_{n} \beta_{n} x_{n}=\Sigma\left|\alpha_{n}\right| x_{n}=|x|$.

The other part is proved similarly.

Now, let $x \in A, x=\Sigma \alpha_{n} x_{n}$. If $E$ is any subset of $\mathrm{N}$, then we define $x_{E}$ by $x_{E}=\sum_{n=1}^{\infty} \chi_{E}(n) \alpha_{n} x_{n}$, where $\chi_{E}$ is the characteristic function on $E$. We note that since the basis is unconditional (hence subseries convergent) it follows that $x_{E}$ exists in $A$ for each $x \in A$ and for each subset $E$ of $\mathbf{N}$. If $E \neq \varnothing$ we will call an element $x \in A E$-regular if there exists $y \in A$ such that $x y=e_{E}$ and if $E=\varnothing$ then by $x E$-regular we mean $x$ is singular. If $x$ is not $E$-regular then we call $x$ $E$-singular. We give the following characterization of $E$-regular elements in terms of principal ideals.

Proposition 1. $x$ is E-regular if and only if the principal ideal $\left\langle x_{E}\right\rangle$ generated by $x_{E}$ is closed.

ProOp. If $x$ is $E$-regular then $x_{n}^{*}\left(x_{E}\right) \neq 0$ for every $n \in E$, and there exists $y \in A$ such that $x y=e_{E}$ and so $e_{E} \in\left\langle x_{E}\right\rangle$. If $z \in A$, then $z e_{E}=z_{E} \in\left\langle x_{E}\right\rangle$ and so $\left\langle x_{E}\right\rangle$ contains all elements $z \in A$ for which $x_{n}^{*}(z)=0$ for $n \in E^{c}$ (the complement of $E)$. It follows that $\left\langle x_{E}\right\rangle=\cap\left\{M_{k}: k \in E^{c}\right\}$, where $M_{k}=\left\{x \in A \mid x_{k}^{*}(x)=0\right\}$ is a closed maximal ideal [3, Theorem 2.1]. Thus $\left\langle x_{E}\right\rangle$ is closed.

Conversely, if $x_{E}$ is closed, then by [3, Corollary 2.3], $\left\langle x_{E}\right\rangle=\cap\left\{M_{k}: k \in E^{c}\right\}$ and hence $e_{E} \in\left\langle x_{E}\right\rangle$. Therefore, there exists $y \in A$ such that $y x_{E}=e_{E}$ and this shows that $x$ is $E$-regular.

We now state some elementary properties about $E$-regular elements which we will need later. The proofs follow directly from the definition so we will not give all the details.

LEMMA 3. (a) If $x$ is $E$-regular and $F \subseteq E$, then $x$ is $F$-regular.

(b) If $x$ is both $E$-regular and $F$-regular, then $x$ is $E \cup F$-regular.

(c) If $x$ is $E$-regular, then $|x|$ is also E-regular.

(d) If $\left|x_{n}^{*}(x)\right|<\left|x_{n}^{*}(y)\right|(n>1)$ and $x$ is E-regular, then $y$ is E-regular.

(e) If $x$ is $E$-regular and $y$ is $F$-regular, then $x y$ is $E \cap F$-regular.

(f) If $x$ is E-regular and $y$ is F-regular, then $|x|+|y|$ is $E \cup F$-regular. 
Proof. We will only prove (f). For this, note that for $n \in N,\left|x_{n n}^{*}(x)\right|+\left|x_{n}^{*}(y)\right|$ $>\left|x_{n n}^{*}(x)\right|$; i.e. $\left|x_{n}^{*}(|x|+|y|)\right|>\left|x_{n}^{*}(x)\right|$. From (d) it follows that $|x|+|y|$ is $E$-regular and similarly $|x|+|y|$ is $F$-regular. Hence the result follows by (b).

We aim to associate a filter to each ideal of $A$. For this we define a function $F$ from $A$ to $\mathscr{P}(\mathscr{P}(\mathbf{N}))$ by $\mathbf{F}(x)=\left\{E \subseteq \mathbf{N} \mid x\right.$ is $E^{c}$-regular $\}$ and for a subset $I$ of $A$ we set $\mathbf{F}[I]=\cup_{x \in I} F(x)$.

LEMMA 4. (a) $\mathbf{F}(x y) \subseteq \mathbf{F}(x) \cap \mathbf{F}(y)$.

(b) $\mathbf{F}(|x|+|y|) \supseteq \mathbf{F}(x) \cup \mathbf{F}(y)$.

(c) If $\left|x_{n}^{*}(x)\right|<\left|x_{n}^{*}(y)\right|(n>1)$, then $\mathbf{F}(x) \subseteq \mathbf{F}(y)$.

Proof. (a) If $x y$ is $E^{c}$-regular then it is clear that both $x$ and $y$ are also $E^{c}$-regular.

(b) If $x$ is $E^{c}$-regular then by Lemma $3\left(\right.$ a) and (f), $|x|+|y|$ is also $E^{c}$-regular. Since the situation is symmetric with respect to $x$ and $y$ the result follows.

(c) This follows from Lemma 3(d).

Proposition 2. $\mathbf{F}(x)$ is a filter on $\mathbf{N}$ if and only if $x$ is noninvertible.

Proof. If $x$ is invertible then clearly $\varnothing \in \mathbf{F}[x]$ and thus $F(x)$ is not a filter. Conversely, if $x$ is noninvertible then $\varnothing \notin \mathrm{F}(x)$. If $E, F \in \mathrm{F}(x)$, then $x$ is $E^{c}$-regular and $F^{c}$-regular; hence, by Lemma $3(\mathrm{~b}), x$ is $E^{c} \cup F^{c}$-regular. Now $E^{c} \cup F^{c}=$ $(E \cap F)^{c}$ and so $E \cap F \in F(x)$. Finally, if $E \in F(x)$ and $F \supseteq E$ (i.e. $F^{c} \subseteq E^{c}$ ) then it follows from the fact that $x$ is $E^{c}$-regular and by Lemma 3(a) that $x$ is $F^{c}$-regular. Hence $F \in F(x)$.

Proposition 3. If $I$ is an ideal (maximal ideal), then $\mathbf{F}[I]$ is a filter (ultrafilter).

Proor. It follows by Proposition 2 that $\varnothing \notin \mathbf{F}(x)$ for each $x \in I$ and so $\varnothing \notin \mathrm{F}[I]$. If $E, F \in \mathrm{F}[I]$ then there exist $x, y \in I$ such that $x$ is $E^{c}$-regular and $y$ is $F^{c}$-regular. By Lemma $2, w=|x|+|y| \in I$ and by Lemma 3(f), $w$ is $E^{c} \cup F^{c}=$ $(E \cap F)^{c}$-regular whence $E \cap F \in \mathbf{F}[I]$. Finally, if $E \in \mathbf{F}[I]$ then $E \in F(x)$ for some $x \in I$ and hence if $F \supseteq E, F \in F(x)$ by Proposition 2 whence $F \in F[I]$. This shows that $F[I]$ is a filter.

Suppose $I$ is maximal and $G$ is any subset of $N$ such that $G \cap F \neq \varnothing$ for every $F \in \mathrm{F}[I]$. Let $H=G^{c}$ and suppose that $e_{H} \notin I$; then $e \in I+\left\langle e_{H}\right\rangle$; i.e. there exist $y \in I$ and $z \in A$ such that $e=y+z e_{H}$. Hence $y=e-z e_{H}$, so that $y e_{G}=$ $e e_{G}-z e_{H} e_{G}=e_{G}$. Therefore $e_{G} \in I$ and since $H \in F\left(e_{G}\right)$, it follows that $H \in$ $F[I]$, contradicting the fact that $G \cap F \neq \varnothing$ for every $F \in F[I]$. This shows that $e_{H} \in I$ and so $G \in \mathrm{F}[I]$. Thus $\mathrm{F}[I]$ is an ultrafilter.

We now wish to show that the function $F$ is one-one from the set of maximal ideals of $A$ to the set of ultrafilters on $\mathbf{N}$. For this we define the inverse function of $\mathbf{F}$ by $\bar{F}[\mathcal{F}]=\{x \in A \mid \mathbf{F}(x) \subseteq \mathscr{F}\}$ for a filter $\mathscr{F}$ on $\mathbf{N}$. Observe that we have shown that the set $M(A)$ of closed maximal ideals of $A$ is equal to $\left\{x_{n}^{*} \mid n \in N\right\}$. We identify $M(A)$ with $\mathbf{N}$ via the map $x_{n}^{*} \leftrightarrow n$ and consider the Gelfand transform $\hat{x}$ of an element $x \in A$ to be the function on $N$ given by $\hat{x}(n)=x_{n}^{*}(x)$. We need the following two lemmas: 
Lemma 5. If $x \in A$ and $E$ is a subset of $\mathbf{N}$ such that $E \cap F \neq \varnothing$ for every $F \in \mathbf{F}(x)$, then $x$ is $E$-singular.

Proof. If $x$ is $E$-regular then $x$ is $\left(E^{c}\right)^{c}$-regular and thus $E^{c} \in \mathbf{F}(x)$. However, $E \cap E^{c}=\varnothing$ contradicting the hypothesis of the lemma.

Lemma 6. Let $x \in A$ and let $\mathscr{F}$ be an ultrafilter base on $\mathrm{N}$. If $\lim _{\mathscr{G}} x z=0$ for every $z \in A$ then $x$ is $E$-singular for every $E \in \mathscr{F}$.

Proof. If $x$ is $E$-regular for some $E \in \mathscr{F}$ then there exists $z \in A$ such that $x z=e_{E}$ and hence 1 is a cluster point of $\widehat{x z}(\mathscr{F})$ [because $E \cap F \neq \varnothing$ and so $1 \in \widehat{x z}(\mathscr{F})$ for each $F \in \mathscr{F}]$. But 0 is also a cluster point of $\widehat{x z}(\mathscr{F})$ by hypothesis, contradicting the fact that $\widehat{x z}(\mathscr{F})$ is an ultrafilter base.

PRoposition 4. If $\mathscr{F}$ is an ultrafilter on $\mathbf{N}$ then $\overline{\mathbf{F}}[\mathcal{F}]$ is a maximal ideal.

Proof. Let $M=\overleftarrow{\mathbf{F}}[\mathscr{F}]$. If $x \in M$ then $\mathbf{F}(x) \subseteq \mathscr{F}$ and so $x$ is noninvertible by Proposition 2. Thus $M$ consists of noninvertible elements only. If $x \in M$ and $y \in A$ then, by Lemma $4(a), F(x y) \subseteq F(x)$ whence $x y \in M$. Finally, if $x, y \in M$ then $\mathbf{F}(x) \subseteq \mathscr{F}$ and $\mathbf{F}(y) \subseteq \mathscr{F}$; hence by Lemma $5, x$ and $y$ are $E$-singular for every $E \in \mathscr{F}$. Thus for $z \in A, x z$ and $y z$ are also $E$-singular for every $E \in \mathscr{F}$. By Lemma 1(b) (applied only on $E$ ) we have that $\inf \{\widehat{x z}(n) ; n \in E\}=0$ for all $E \in \mathscr{F}$ and thus 0 is a cluster point of the filter base $\widehat{x z}(\mathscr{F})$. Similarly 0 is a cluster point of the filter base $\widehat{y z}(\mathscr{F})$. Since $\mathscr{F}$ is an ultrafilter, $\widehat{x z}(\mathscr{F})$ and $\widehat{y z}(\mathscr{F})$ are ultrafilter bases on $C$ and hence both converge to 0 for every $z \in A$. From the continuity of addition in $C$ we have $\lim _{\mathscr{F}}\left(\widehat{x+y) z}=\lim _{\mathscr{F}}(\widehat{x z}+\widehat{y z})=\lim _{\mathscr{F}} \widehat{x z}+\right.$ $\lim _{\mathscr{f}} \widehat{y z}=0$ for all $z \in A$. From this and Lemma 6 we conclude that $x+y$ is $E$-singular for every $E \in \mathscr{F}$. Therefore $\mathbf{F}(x+y) \subseteq \mathscr{F}$ and consequently $x+y \in$ $M$. This shows that $M$ is an ideal. Now, suppose that $N$ is an ideal in $A$ containing $M$. Then $F[N] \supseteq \mathscr{F}$ and since $\mathscr{F}$ is an ultrafilter $F[N]=\mathscr{F}$. So, if $x \in N \backslash M$ then $F(x) \subseteq \mathscr{F}$ and so $x \in M$ by definition. Thus $M=N$ and this shows that $M$ is maximal.

Propostrion 5. For $\mathscr{F}$ an ultrafilter on $\mathbf{N}$ and for $M$ a maximal ideal in $A$ the following relations hold: $\mathbf{F}[\overline{\mathrm{F}}[\mathcal{F}]]=\mathscr{F}$ and $\overline{\mathrm{F}}[\mathrm{F}[M]]=M$.

Proof. To prove the first relation let $N=\overline{\mathrm{F}}[\mathscr{F}]$. By Proposition $4, N$ is a maximal ideal and clearly $\mathbf{F}[N] \supseteq \mathscr{F}$. But since $\mathscr{F}$ is an ultrafilter the result follows. The second relation is proved similarly.

This proposition shows that the map: $M \rightarrow F[M]$ is one-one from the set $M^{\#}(A)$ onto the set of ultrafilters on $\mathbf{N}$.

We recall here that if $\mathfrak{A}$ is the collection of all ultrafilters on $\mathbf{N}$ then the sets $E^{*}=\{\mathscr{F} \in \mathfrak{X}: E \in \mathscr{F}\}, E \subseteq \mathbf{N}$, form a base for the closed sets for a topology on $\mathfrak{A}$ and in this topology $\mathfrak{A}$ is $\boldsymbol{\beta} \mathbf{N}$, the Stone-Čech compactification of $\mathbf{N}$. Also, there is a natural topology on the set $\mathbf{M}^{\#}(A)$-the hull-kernel topology [G]. A base for the closed sets in this topology is given by the family of sets of the form $H(x)=\{M \in$ $\left.M^{\#}(A): x \in M\right\}, x \in A$. However, for our algebras we show that a much smaller collection forms a base. 
LEMMA 7. The sets of the form $H\left(e_{E}\right), E \subseteq N$, are a base for the closed sets in the hull-kernel topology on $\mathbf{M}^{\#}(A)$.

Proof. We show that for each noninvertible $x \in A, H(x)=\cap\left\{H\left(e_{E}\right): x\right.$ is $E$ regular \}. To this end note that if $M \in H(x)$ (i.e. $x \in M$ ) and if $x$ is $E$-regular then there exists $z \in A$ such that $x z=e_{E}$ and thus $e_{E} \in M$. It follows that $M \in H\left(e_{E}\right)$ and thus $M \in \cap\left\{H\left(e_{E}\right): x\right.$ is $E$-regular $\}$. Conversely, if $M \in$ $\cap\left\{H\left(e_{E}\right): x\right.$ is $E$-regular $\}$, then clearly $F(x) \subseteq F[M]$ and thus $x \in \bar{F}[F[M]]$. Since $M$ is a maximal ideal it follows by Proposition 5 that $\mathbf{F}[\mathbf{F}[M]]=M$ and so $x \in M$, i.e. $M \in H(x)$.

We now prove the main result of this section:

THEOREM 1. The map $M \rightarrow F[M]$ is a homeomorphism from $\mathbf{M}^{\#}(A)$ with the hull-kernel topology onto $\beta \mathbf{N}$, the Stone-Čech compactification of $\mathbf{N}$.

Proof. We have already shown that this map, call it $\psi$, is one-one and onto. We will show that $\psi$ maps the base for the closed sets in $\mathbf{M}^{\#}(A)$ onto the base for the closed sets in $\beta N$ and this will show that $\psi$ is a homeomorphism. To this end note that if $E \subseteq N$ and $M \in H\left(e_{E}\right)$ then $E^{c} \in F[M]$. Conversely, if $E^{c} \in F[M]$, then there exists $x \in M$ such that $x$ is $E$-regular and therefore (as in the proof of Lemma 7) $e_{E} \in M$. Thus $\psi$ takes $H\left(e_{E}\right)$ to $\left(E^{c}\right)^{c}$ and this concludes the proof.

We have shown in [3, Theorem 2.1] that each closed maximal ideal of $A$ is of the form $M_{k}=\left\{x \in A: x_{k}^{*}(x)=0\right\}$. It follows that $F\left[M_{k}\right]$ is the fixed ultrafilter [2] consisting of all subsets of $\mathbf{N}$ containing $k$. It follows also that the dense maximal ideals of $A$ correspond under $\mathbf{F}$ to the free ultrafilters on $\mathbf{N}$. We use these facts in the next section.

2. Dense maximal ideals and the dual space. We begin by describing the ideal which is the intersection of the dense maximal ideals of $A$. To this end, set $J(A)=\left\{x \in A: \lim _{n \rightarrow \infty} x_{n}^{*}(x y)=0\right.$ for all $\left.y \in A\right\}$. Evidently $J(A)$ is an ideal of $A$ and clearly it is dense in $A$ because it contains all elements of the form $x=$ $\sum_{i=1}^{n} \alpha_{i} x_{i}$.

LEMMA 8. $x \in J(A)$ if and only if $x$ is not $E$-regular for every infinite $E \subseteq N$.

Proof. If there exists an infinite subset $E$ of $\mathbf{N}$ for which $x$ is $E$-regular then there exists $y \in A$ such that $x y=e_{E}$. But then $\lim _{n \rightarrow \infty} x_{n}^{*}(x y)$ is clearly not 0 and so $x \notin J(A)$.

Conversely, if $x \notin J(A)$ then there exists $y \in A$ such that $\lim _{n \rightarrow \infty} x_{n}^{*}(x y)$ is not 0 . So, let $r=\varlimsup|\lim | x_{n}^{*}(x y) \mid, r \neq 0$. Thus there exists a subsequence $\left\{n_{k}\right\}$ of $\mathbf{N}$ such that $\lim _{k \rightarrow \infty}\left|x_{n_{k}}^{*}(x y)\right|=r$, and without loss of generality we can choose $\left\{n_{k}\right\}$ in such a way that $x_{n_{k}}^{*}(x y) \neq 0$ for all $k \geqslant 1$ (because $x_{n_{k}}^{*}(x y)=0$ can happen only finitely often). It follows by Lemma 1(b) (applied on $\left\{n_{k}\right\}$ ) that $x y$ is $\left\{n_{k}\right\}$-regular and so $x$ is also $\left\{n_{k}\right\}$-regular; i.e., $x$ is $E$-regular on the infinite set $E=\left\{n_{k}\right\}$.

TheOREM 2. $J(A)=\bigcap\left\{M: M \in \mathbf{M}^{\#}(A) \backslash \mathbf{M}(A)\right\}$. 
Proof. " $\subseteq$ ". For this fix $x \in J(A)$ and $M \in M^{\#}(A) \backslash M(A)$. Let $\mathscr{F}=\overline{\mathbf{F}}[M]$. We show that $\mathbf{F}(x) \subseteq \mathcal{F}$. Let $E \in \mathbf{F}(x)$. Then since $\lim _{\mathscr{F}} \widehat{x z}=0$ for all $z \in A$, we see that $x$ is $F$-singular for all $F \in \mathcal{F}$. If $E \notin \mathcal{F}$, then $E^{c} \in \mathcal{F}$ and so $x$ would be $E^{c}$-singular which is contrary to the fact that $E \in \mathrm{F}(x)$.

Conversely, suppose $x \in \cap\left\{M: M \in \mathbf{M}^{\#}(A) \backslash M(A)\right\}$. Let $E$ be an infinite subset of $\mathbf{N}$ and let $\mathscr{F}$ be a free ultrafilter with $E \in \mathscr{F}$. If $x$ is $E$-regular then $E^{c} \in \mathbf{F}(x)$ and since $E \in \mathscr{F}$ it follows that $\mathbf{F}(x) \not \mathcal{F}$. This implies that $x \notin \overleftarrow{F}[\mathcal{F}]$ which is a dense maximal ideal. It follows that $x \notin \cap\left\{M: M \in M^{\#}(A) \backslash M(A)\right\}$ contradicting our hypothesis. This contradiction stems from the assumption that $x$ is $E$-regular on an infinite set $E$. It follows that $x$ is not $E$-regular for any infinite $E$ and so, by Lemma $8, x \in J(A)$.

To study the dual space we define a subset of $A, K(A)=\left\{x \in A: \sum_{n-1}^{\infty}\left|x_{n}^{*}(x y)\right|\right.$ $<\infty$ for all $y \in A\}$. It is easy to see that $K(A)$ is an ideal and it is dense because all finite linear combinations of elements from $\left\{x_{n}\right\}$ belong to it. Also, note that $K(A) \subseteq J(A)$ so that $\overline{K(A)}=\overline{J(A)}=A$.

For the next theorem we need the additional hypothesis that $A$ be metrizable; i.e. $\boldsymbol{A}$ is a complete metrizable LC-algebra. Algebras of this type are called $\boldsymbol{B}_{\mathbf{0}}$-algebras.

THEOREM 3. If $A$ is a $B_{0}$-algebra with identity and if $A$ has an unconditional orthogonal basis $\left\{x_{n}\right\}$ then $A^{\prime}$ is linearly isomorphic to the ideal $K(A)$.

Proof. Consider the map $L: K(A) \rightarrow A^{\prime}$ defined by $y \rightarrow f_{y}$ where $f_{y}(x)=$ $\sum_{n=1}^{\infty} x_{n}^{*}(x y)$. Now, set $f_{y}^{k}(x)=\sum_{n-1}^{k} x_{n}^{*}(x y)=\sum_{n-1}^{k} x_{n}^{*}(x) x_{n}^{*}(y)$. Clearly each $f_{y}^{k}$ is continuous (since it is a finite linear combination of the continuous functionals $x_{k}^{*}$ ) and $\lim _{k \rightarrow \infty} f_{y}^{k}(x)=f_{y}(x)$ for $x \in A$. Hence by the Banach-Steinhaus theorem [1], $f_{y} \in A^{\prime}$.

Now, if $y_{1}, y_{2} \in K(A), y_{1} \neq y_{2}$, then there exists $n>1$ such that $x_{n}^{*}\left(y_{1}\right) \neq x_{n}^{*}\left(y_{2}\right)$ and since $f_{y_{1}}\left(x_{n}\right)=x_{n}^{*}\left(y_{1}\right)$ and $f_{y_{2}}\left(x_{n}\right)=x_{n}^{*}\left(y_{2}\right)$ it follows that $f_{y_{1}} \neq f_{y_{2}}$. Thus $L$ is one-one.

Finally, suppose $f \in A^{\prime}$ and set $\alpha_{n}=f\left(x_{n}\right), n>1$. Since $e=\sum_{n=1}^{\infty} x_{n}$ (cf. [3]), $f(e)=\sum_{n-1}^{\infty} f\left(x_{n}\right)=\sum_{n=1}^{\infty} \alpha_{n}$ and this series converges. Therefore $\left\{\alpha_{n}\right\}$ is a bounded sequence and thus, by Lemma $1(\mathrm{a}), w=\sum_{n=1}^{\infty} \alpha_{n} x_{n} \in A$. For $x \in A, f(x)=$ $\sum_{n=1}^{\infty} x_{n}^{*}(x) f\left(x_{n}\right)=\sum_{n=1}^{\infty} x_{n}^{*}(x) \alpha_{n}=\sum_{n=1}^{\infty} x_{n}^{*}(x) x_{n}^{*}(w)=\sum_{n=1}^{\infty} x_{n}^{*}(x w)$ and since this series converges it follows that $w \in K(A)$. Clearly $f=f_{w}$ and this shows that $L$ is onto $A^{\prime}$.

We note that in general $K(A) \neq J(A)$. Thanks to the referee for the following example:

Let $w_{n}=\left\{w_{n_{k}}\right\}_{k>1} \in \mathbf{R}^{\mathbf{N}}$ where $w_{n_{k}}=k^{-1 / 2^{n}}$ for $n>0$ and let $A=\left\{x=\left\{x_{k}\right\}\right.$ : $\lim _{k} x_{k} w_{m_{k}}=0$ for $\left.n>0\right\}$. With $\|x\|_{n}=\sup _{k}\left|x_{k}\right| w_{n_{k}}(n>0)$ as seminorms, $A$ is a $B_{0}$-algebra having identity $e=\{1\}$ and an orthogonal unconditional basis $\left\{e_{n}\right\}, e_{n}$ $=\left\{\delta_{n m}\right\}_{m>1},\left(\delta_{n m}\right.$ is the Kronecker delta). From definition it is clear that $w_{0} \in$ $J(A)$ but $w_{0} \notin K(A)$ because $w_{0}=\{1 / k\}$ is not summable.

However, for the algebra of all complex sequences and for the algebra $H(D)$ of all holomorphic functions on the open unit disc $D$ with the Hadamard product (see Examples 1 and 4 of [3]) we have that $K(A)=J(A)$. Thus in these cases the dual 
space is linearly isomorphic to the intersection of the dense maximal ideals. We give below a condition that guarantees the equality $K(A)=J(A)$. First, we note that $B_{0}$-algebras with identity having unconditional orthogonal bases always contain elements $x$ such that the sequence $\left\{x_{n}^{*}(x)\right\}$ is unbounded. For if not, then a simple application of the closed graph theorem shows that the map $x \rightarrow\left\{x_{n}^{*}(x)\right\}$ of $A$ to $\boldsymbol{m}$ (the Banach space of bounded sequences) is a topological isomorphism. But this is impossible since $m$ is not separable. We require a slightly stronger condition than the existence of elements with unbounded coefficients to show that $K(A)=$ $J(A)$ in the following:

Proposition 6. If there is an invertible element $x \in A$ such that $\sum_{n=1}^{\infty}\left|x_{n}^{*}(x)\right|<$ $\infty$, then $K(A)=J(A)$.

Proof. Let $x$ be as in the statement and let $y \in J(A)$. Then for every $z \in$ $A, \lim _{n \rightarrow \infty} x_{n}^{*}\left(y z x^{-1}\right)=0$. Since $x^{-1}=\sum_{n=1}^{\infty} x_{n}^{*}(x)^{-1} x_{n}$ it follows that $\lim _{n \rightarrow \infty} x_{n}^{*}(y z) x_{n}^{*}(x)^{-1}=0$. Hence for $n$ (sufficiently large) $\left|x_{n}^{*}(y z) x_{n}^{*}(x)^{-1}\right|<1$; i.e. $\left|x_{n}^{*}(y z)\right|<\left|x_{n}^{*}(x)\right|, z \in A$, and since $\sum_{n-1}^{\infty}\left|x_{n}^{*}(x)\right|<\infty$ it follows that the series $\sum_{n=1}^{\infty} x_{n}^{*}(y z)$ converges for $z \in A$. Thus $y \in K(A)$, i.e. $J(A) \subseteq K(A)$. The reverse inclusion being true in general, we have proved the proposition.

\section{REFERENCES}

1. M. M. Day, Normed linear spaces, Academic Press, New York, 1962.

2. L. Gillman and M. Jerison, Rings of continuous functions, Van Nostrand, Princeton, N. J., 1960.

3. T. Husain and S. Watson, Topological algebras with orthogonal bases, Pacific J. Math. (submitted).

4. E. A. Michael, Locally multiplicative convex topological algebras, Mem. Amer. Math. Soc. No. 2, 1952.

5. I. Singer, Bases in Banach spaces. I, Springer-Verlag, New York and Berlin, 1970.

6. W. Zelazko, Banach algebras, Elsevier, New York, 1973.

Department of Mathematical Sciences, McMaster University, Hammion, Ontario, Canada L8S 4K1 